Publ. Mat. 62 (2018), 95-111

DOI: 10.5565 /PUBLMAT6211805

\title{
STABILITY OF GENERALIZED LINEAR WEINGARTEN HYPERSURFACES IMMERSED IN THE EUCLIDEAN SPACE
}

\author{
Jonatan F. da Silva, Henrique F. De Lima, and \\ Marco Antonio L. Velásquez
}

\begin{abstract}
Given a positive function $F$ defined on the unit Euclidean sphere and satisfying a suitable convexity condition, we consider, for hypersurfaces $M^{n}$ immersed in the Euclidean space $\mathbb{R}^{n+1}$, the so-called $k$-th anisotropic mean curvatures $H_{k}^{F}$, $0 \leq k \leq n$. For fixed $0 \leq r \leq s \leq n$, a hypersurface $M^{n}$ of $\mathbb{R}^{n+1}$ is said to be $(r, s, F)$-linear Weingarten when its $k$-th anisotropic mean curvatures $H_{k}^{F}, r \leq k \leq s$, are linearly related. In this setting, we establish the concept of stability concerning closed $(r, s, F)$-linear Weingarten hypersurfaces immersed in $\mathbb{R}^{n+1}$ and, afterwards, we prove that such a hypersurface is stable if, and only if, up to translations and homotheties, it is the Wulff shape of $F$. For $r=s$ and $F \equiv 1$, our results amount to the standard stability studied, for instance, by Alencar-do Carmo-Rosenberg [1] .
\end{abstract}

2010 Mathematics Subject Classification: Primary: 53C42; Secondary: 53B25.

Key words: Euclidean space, Wulff shape, $k$-th anisotropic mean curvatures, $(r, s, F)$-linear Weingarten hypersurfaces, stable closed hypersurfaces.

\section{Introduction and statement of the main result}

In recent years, following the seminal ideas established by Reilly in $[\mathbf{2 4}, \mathbf{2 5}]$, several authors obtained geometric properties of a hypersurface through the study of the Euler-Lagrange equation associated to certain variational problems (see, for instance, $[\mathbf{1}, 4, \mathbf{5}, \mathbf{6}, \mathbf{1 4}, \mathbf{1 1}, \mathbf{2 9}]$ ). Proceeding into this branch, in this paper we deal with a suitable class of closed hypersurfaces immersed in the Euclidean space $\mathbb{R}^{n+1}$, which are critical points for the variational problem of minimizing a linear combination of certain area functions preserving the volume enclosed. A precise description of our object of study will be given after some preliminaries.

Let $F: \mathbb{S}^{n} \rightarrow \mathbb{R}^{+}$be a positive smooth function which satisfies the following convexity condition:

$$
\left(D^{2} F+F I\right)_{x}>0, \quad \forall x \in \mathbb{S}^{n},
$$


where $D^{2} F$ denotes the intrinsic Hessian of $F$ on the $n$-sphere $\mathbb{S}^{n}$ of $\mathbb{R}^{n+1}$, $I$ denotes the identity on $T_{x} \mathbb{S}^{n}$, and $>0$ means that the matrix is positive definite. We consider the map

$$
\begin{aligned}
\phi: \mathbb{S}^{n} & \longrightarrow \mathbb{R}^{n+1} \\
x & \longmapsto F(x) x+\left(\nabla_{\mathbb{S}^{n}} F\right)_{x},
\end{aligned}
$$

whose image $\mathcal{W}_{F}=\phi\left(\mathbb{S}^{n}\right)$ is a smooth, convex hypersurfaces in $\mathbb{R}^{n+1}$ called the Wulff shape of $F$ (for more details concerning the properties of the Wulff shape see, for instance, $[\mathbf{9}, \mathbf{1 4}, \mathbf{1 6}, \mathbf{1 7}, \mathbf{1 8}, \mathbf{1 9}, \mathbf{2 7}])$. We note that, when $F \equiv 1$ we have that the Wulff shape of $F$ is just the $n$-dimensional Euclidean sphere $\mathbb{S}^{n} \subset \mathbb{R}^{n+1}$.

Throughout this paper, $x: M^{n} \hookrightarrow \mathbb{R}^{n+1}$ will stand for a smooth immersion of a closed oriented hypersurface and $N: M^{n} \rightarrow \mathbb{S}^{n}$ will denote its corresponding Gauss map. In this setting, let

$$
A_{F}:=D^{2} F+F I
$$

and

$$
N_{F}:=\phi \circ N: M^{n} \longrightarrow \mathcal{W}_{F},
$$

which is called the generalized Gauss map into the Wulff shape. Then

$$
S_{F}:=-d N_{F}=-A_{F} \circ d N
$$

is defined as being the $F$-Weingarten operator.

According to the previous definitions, we note that although $A_{F}$ and $d N$ be symmetric operators, $S_{F}$ is symmetric if and only if $A_{F}$ and $d N$ commute, which does not occur in general. We point out that all the roots of the characteristic polynomial of $S_{F}$ are real. The eigenvalues of $S_{F}$ are called the anisotropic principal curvatures of $x$ and are denoted by $\lambda_{1}, \ldots, \lambda_{n}$. Moreover, if the principal curvatures of $x$ are positive, so are the anisotropic principal curvatures $\lambda_{i}$ (for a proof, see Lemma 2 in $[\mathbf{1 0}])$.

At each $p \in M^{n}, S_{F}$ restricts to a linear map $S_{F}(p): T_{p} M \rightarrow T_{p} M$. For $1 \leq k \leq n$, let $S_{k}(p)$ denote the $k$-th elementary symmetric function on the eigenvalues of $S_{F}(p)$; thus one gets $n$ smooth functions $S_{k}: M \rightarrow$ $\mathbb{R}$, such that

$$
\operatorname{det}\left(t I-S_{F}\right)=\sum_{k=0}^{n}(-1)^{k} S_{k} t^{n-k},
$$

where $S_{0} \equiv 1$ by construction. If $p \in M^{n}$ and $\left\{\lambda_{k}\right\}$ are the eigenvalues with respect to the operator $S_{F}(p)$, one immediately sees that

$$
S_{k}=\sigma_{k}\left(\lambda_{1}, \ldots, \lambda_{n}\right):=\sum_{1 \leq i_{1}<\cdots<i_{k} \leq n} \lambda_{i_{1}} \cdots \lambda_{i_{k}},
$$


where $\sigma_{k} \in \mathbb{R}\left[X_{1}, \ldots, X_{n}\right]$ is the $k$-th elementary symmetric polynomial on the indeterminates $X_{1}, \ldots, X_{n}$.

For $1 \leq k \leq n$, one defines the $k$-th anisotropic mean curvature $H_{k}^{F}$ of $x$ by

$$
\left(\begin{array}{l}
n \\
k
\end{array}\right) H_{k}^{F}=\sigma_{k}\left(\lambda_{1}, \ldots, \lambda_{n}\right) .
$$

There exist many works concerning the properties of the $k$-th anisotropic mean curvatures of hypersurfaces in $\mathbb{R}^{n+1}$ space. We refer the readers to $[10,13,14,15,16,23,30]$.

At this point, we are in a position to define our geometrical object of study: an immersion $x: M^{n} \hookrightarrow \mathbb{R}^{n+1}$ is said a $(r, s, F)$-linear Weingarten hypersurface if, for some integers $r$ and $s$ satisfying the inequality $0 \leq$ $r \leq s \leq n-1$, holds the following linear relation

$$
a_{r} b_{r} H_{r+1}^{F}+\cdots+a_{s} b_{s} H_{s+1}^{F}=\text { constant, }
$$

for some nonnegative real numbers $a_{k}, k \in\{r, \ldots, s\}$, with at least one non zero, where $b_{k}=(k+1)\left(\begin{array}{c}n \\ k+1\end{array}\right)$ and $F: \mathbb{S}^{n} \rightarrow \mathbb{R}^{+}$is a positive smooth function which satisfies the convexity condition (1.1).

We observe that, when $r=0, s=1$, and $F=1$, these hypersurfaces are classically called linear Weingarten hypersurfaces and, in the last years, a vast literature has been produced in the direction to obtain characterization results of them (see, for instance, $[2,3,7,8,20,26]$ ). In this paper we extend this study to the anisotropic case. It is said that a phenomenon has anisotropic behavior when its effects vary with the direction; as opposed to isotropic behavior (homogeneous in all directions). This is observed in many phenomena of nature. Indeed, it appears that anisotropic properties are present in the study of many phenomena. For example, crystals whose propagation of light depends on the direction exhibit optical anisotropy [28]. The $(s, s, F)$-linear Weingarten hypersurfaces are exactly the hypersurfaces with $H_{s+1}^{F}$ constant. On the other hand, taking into account that all the anisotropic principal curvatures of the Wulff shape $\mathcal{W}_{F}$ are constant (see, for example, [14]), we have that $\mathcal{W}_{F}$ constitutes a natural example of closed $(r, s, F)$-linear Weingarten hypersurface immersed in $\mathbb{R}^{n+1}$, for any $0 \leq r \leq s \leq n-1$.

Motivated by the previous discussion, here we will establish the notion of stability concerning closed $(r, s, F)$-linear Weingarten hypersurfaces in $\mathbb{R}^{n+1}$. Such concept arises considering the variational problem of minimizing a suitable linear combination of certain functionals $(k, F)$-th areas for volume-preserving variations (cf. Section 4). Now, we are in position to state our main result. 
Theorem 1. Let $r$ and $s$ be integers satisfying $0 \leq r \leq s \leq n-2$, $n \geq 3$, and let $x: M^{n} \hookrightarrow \mathbb{R}^{n+1}$ be a closed $(r, s, F)$-linear Weingarten hypersurface with $H_{s+1}^{F}$ positive. Then, $x: M^{n} \hookrightarrow \mathbb{R}^{n+1}$ is stable if, and only if, up to translations and homotheties, $x(M)$ is the Wulff shape of $F$.

We observed that taking $r=s$ in Theorem 1 we reobtain Theorem 1.3 of [14]. The proof of Theorem 1 is given in Section 5 .

Remark 1. Related to the isotropic case, Micallef and Moore [22] proved that any minimal 2-sphere in a manifold with positive isotropic curvature is unstable. More recently, $\mathrm{Li}[\mathbf{2 1}]$ showed the nonexistence of stable immersed minimal surfaces uniformly conformally equivalent to $\mathbb{C}$ in any complete orientable 4-dimensional Riemannian manifold with uniformly positive isotropic curvature.

\section{The operators $P_{k}, T_{k}$, and $L_{k}$}

In order to give a description of our variational problem, we will need to define some suitable operators associated to a hypersurface $x: M^{n} \hookrightarrow \mathbb{R}^{n+1}$ as in the previous section. The first ones are the operators $P_{k}: \mathfrak{X}(M) \rightarrow \mathfrak{X}(M), 0 \leq k \leq n$, which can be defined inductively from the $F$-Weingarten operator $S_{F}$ and $k$-th anisotropic mean curvatures $H_{k}^{F}$ by

$$
P_{0}=I \quad \text { and } \quad P_{k}=\left(\begin{array}{l}
n \\
k
\end{array}\right) H_{k}^{F} I-P_{k-1} \circ S_{F},
$$

where $I$ denotes the identity in $\mathfrak{X}(M)$. Equivalently,

$$
P_{k}=\sum_{j=0}^{k}(-1)^{j}\left(\begin{array}{c}
n \\
k-j
\end{array}\right) H_{k-j}^{F} S_{F}^{j} .
$$

Note that each $P_{k}(p)$ is also a linear operator on each tangent space $T_{p} M$ which commutes with $S_{F}(p)$.

The operator $T_{k}: \mathfrak{X}(M) \rightarrow \mathfrak{X}(M), 0 \leq k \leq n$, is defined by

$$
T_{k}=P_{k} \circ A_{F} .
$$

Note that, since $A_{F}$ and $d N$ are symmetric, from (2.2) we have that the transformations $T_{k}$ are all self-adjoint operators. So, likewise $S_{F} \circ A F$, $d N \circ S_{F}$, and $d N \circ P_{k}$ are symmetric. Moreover, taking into account that

$$
T_{k-1} \circ d N=P_{k-1} \circ A_{F} \circ d N=-P_{k-1} \circ S_{F},
$$


we get

$$
P_{k}=\left(\begin{array}{l}
n \\
k
\end{array}\right) H_{k}^{F} I-P_{k-1} \circ S_{F}=\left(\begin{array}{l}
n \\
k
\end{array}\right) H_{k}^{F} I+T_{k-1} \circ d N .
$$

Finally, we define the operator $L_{k}: \mathcal{C}^{\infty}(M) \rightarrow \mathcal{C}^{\infty}(M)$ by

$$
L_{k}(f)=\operatorname{div}\left(T_{k}(\nabla f)\right) \text {. }
$$

Equivalently,

$$
L_{k}(f)=\sum_{i, j}\left[\left(T_{k}\right)_{i j} f_{j}\right]_{i}
$$

where we denote the coefficients of covariant differential of $f$ and $T_{k}$ with respect to a (local) orthonormal frame $\left\{e_{1}, \ldots, e_{n}\right\}$ on $M^{n}$ by $f_{i}$ and $\left(T_{k}\right)_{i j}$, respectively. In particular, we the $F$-Laplacian is defined by

$$
\Delta_{F}(f):=L_{0}(f)=\operatorname{div}\left(A_{F}(\nabla f)\right) .
$$

\section{Key lemmas}

This section is devoted to other auxiliary results which will be also necessary to prove Theorem 1. Initially, we recall suitable inequalities concerning the $k$-th anisotropic mean curvatures, which will be very useful to show our main result.

Lemma 1. Let $x: M^{n} \hookrightarrow \mathbb{R}^{n+1}$ be a closed hypersurface and $F: \mathbb{S}^{n} \rightarrow$ $\mathbb{R}^{+}$satisfying the condition (1.1). If $H_{s+1}^{F}$ is positive on $M^{n}$ then for $1 \leq k \leq s$, we have:

(i) each $k$-th anisotropic mean curvature $H_{k}^{F}$ is positive;

(ii) $H_{1}^{F} H_{k+1}^{F}-H_{k+2}^{F} \geq 0$.

Moreover equality holds for some $k$ in (ii) if, and only if, the anisotropic principal curvatures are equal.

Proof: Item (i) corresponds to Lemma 10 of [10]. For (ii), it is known that the following generalization of the Cauchy-Schwarz type inequality holds true (see, for instance, [12, Theorem 51, p. 52, and Theorem 144, p. 104]) for any $1 \leq k \leq n-1$ :

$$
\left(H_{k}^{F}\right)^{2}-H_{k-1}^{F} H_{k+1}^{F} \geq 0
$$

the equality occurring for some $k$ if, and only if, at this point the anisotropic principal curvatures are equal. As $H_{k}^{F}>0$ for any $0 \leq$ $k \leq s+1$, we can write the inequality (3.1) as follows:

$$
\frac{H_{k}^{F}}{H_{k-1}^{F}} \geq \frac{H_{k+1}^{F}}{H_{k}^{F}}
$$


for any $1 \leq k \leq s+1$, and equality holds for some $k$ if, and only if, at this point the anisotropic principal curvatures are equal. Hence, we have the inequalities

$$
H_{1}^{F} \geq \frac{H_{2}^{F}}{H_{1}^{F}} \geq \frac{H_{3}^{F}}{H_{2}^{F}} \geq \cdots \geq \frac{H_{k+2}^{F}}{H_{k+1}^{F}},
$$

for all $0 \leq k \leq s$, and equality holds in (3.2) if, and only if, the anisotropic principal curvatures are equal. Thus, from (3.2) we obtain the results, finishing the proof.

Now, we will quote well known result which will be used later (for a proof, see He and Li [13, 15] or Palmer [23]).

Lemma 2. Let $x: M^{n} \hookrightarrow \mathbb{R}^{n+1}$ be an isometric immersion of a closed orientable Riemannian manifold $M^{n}$ and $F: \mathbb{S}^{n} \rightarrow \mathbb{R}^{+}$satisfying the condition (1.1). If $\lambda_{1}=\lambda_{2}=\cdots=\lambda_{n}=$ constant $\neq 0$, then up to translations and homotheties, $x(M)$ is the Wulff shape of $F$.

In the next lemma, we recall the so-called Minkowski formulas (see He and $\mathrm{Li}[\mathbf{1 3}, \mathbf{1 5}])$.

Lemma 3. Let $x: M^{n} \hookrightarrow \mathbb{R}^{n+1}$ be a closed hypersurface and $F: \mathbb{S}^{n} \rightarrow$ $\mathbb{R}^{+}$satisfying the condition (1.1). For each $0 \leq j \leq n-1$, the following Minkowski-type formulas hold

$$
\int_{M}\left(F H_{k}^{F}+H_{k+1}^{F}\langle x, N\rangle\right) d M=0 .
$$

At this point, we will fix some notation. Given $f \in \mathcal{C}^{\infty}(M)$ smooth function, we define:

$$
\begin{aligned}
I_{k}[f] & :=L_{k} f+\left\langle T_{k} d N, d N\right\rangle f ; \\
R_{r, s}[f] & :=\sum_{k=r}^{s}(k+1) a_{k} I_{k}[f] .
\end{aligned}
$$

We will also need of the following result due to $\mathrm{He}$ and $\mathrm{Li}[\mathbf{1 3}]$.

Lemma 4. For each $0 \leq k \leq n-1$, we have:

$$
\begin{aligned}
I_{k}[F(N)]= & -\left(\begin{array}{c}
n \\
k+1
\end{array}\right)\left\langle\nabla H_{k+1}^{F}, \nabla_{S^{n}} F\right\rangle+n\left(\begin{array}{c}
n \\
k+1
\end{array}\right) H_{1}^{F} H_{k+1}^{F} \\
& -(k+2)\left(\begin{array}{c}
n \\
k+2
\end{array}\right) H_{k+2}^{F}
\end{aligned}
$$

$$
I_{k}[\langle x, N\rangle]=-\left(\begin{array}{c}
n \\
k+1
\end{array}\right)\left(\left\langle\nabla H_{k+1}^{F}, x^{\top}\right\rangle-(k+1) H_{k+1}^{F}\right) \text {. }
$$


Finally, we also need of the following symmetry result, which will be crucial to establish the proof of Theorem 1 :

Lemma 5. For $f, g \in \mathcal{C}^{\infty}(M)$ and $0 \leq r \leq s \leq n-1$, we have:

$$
\int_{M} g R_{r, s}[f] d M=\int_{M} f R_{r, s}[g] d M .
$$

Proof: By (3.4) we have

$$
\int_{M} g R_{r, s}[f] d M=\sum_{k=r}^{s}(k+1) a_{k} \int_{M} g I_{k}[f] d M .
$$

By Stokes theorem and from the symmetry of $T_{k}$, we have

$$
\begin{aligned}
\int_{M} g L_{k}(f) d M & =\int_{M} g \operatorname{div} T_{k} \nabla f d M \\
& =-\int_{M}\left\langle T_{k} \nabla f, \nabla g\right\rangle d M \\
& =-\int_{M}\left\langle\nabla f, T_{k} \nabla g\right\rangle d M \\
& =\int_{M} f \operatorname{div} T_{k} \nabla g d M=\int_{M} f L_{k}(g) d M .
\end{aligned}
$$

Thus, by (3.3) and (3.8)

$$
\begin{aligned}
\int_{M} g I_{k}[f] d M & =\int_{M} g\left(L_{k}(f)+\left\langle T_{k} d N, d N\right\rangle f\right) d M \\
& =\int_{M} g L_{k}(f) d M+\int_{M} f g\left\langle T_{k} d N, d N\right\rangle d M \\
& =\int_{M} f L_{k}(g) d M+\int_{M} f g\left\langle T_{k} d N, d N\right\rangle d M \\
& =\int_{M} f\left(L_{k}(g)+\left\langle T_{k} d N, d N\right\rangle g\right) d M=\int_{M} f I_{k}[g] d M .
\end{aligned}
$$

Therefore, by (3.7), (3.9), and (3.4), we have

$$
\int_{M} g R_{r, s}[f] d M=\sum_{k=r}^{s}(k+1) a_{k} \int_{M} f I_{k}[g] d M=\int_{M} f R_{r, s}[g] d M,
$$

finishing the proof. 


\section{Description of the variational problem}

A variation of a closed oriented hypersurface $x: M^{n} \hookrightarrow \mathbb{R}^{n+1}$ is a smooth map

$$
X: M^{n} \times(-\epsilon, \epsilon) \longrightarrow \mathbb{R}^{n+1}
$$

such that, for all $t \in(-\epsilon, \epsilon)$, the map $X_{t}: M^{n} \rightarrow \mathbb{R}^{n+1}$ given by $X_{t}(p)=$ $X(t, p)$ is an immersion such that $X_{0}=x$. In what follows, $d M_{t}$ will denote the volume element of the metric induced on $M^{n}$ by $X_{t}$ and $N_{t}$ will stand for the unit normal vector field along $X_{t}$.

The variational field associated to the variation $X$ is the vector field $\left.\frac{\partial X}{\partial t}\right|_{t=0}$. In this setting, denoting by $(\cdot)^{\top}$ the tangential component on $M^{\bar{n}}$, we have that

$$
\frac{\partial X}{\partial t}=f N_{t}+\left(\frac{\partial X}{\partial t}\right)^{\top}
$$

where

$$
f=\left\langle\frac{\partial X}{\partial t}, N_{t}\right\rangle
$$

The volume of the variation $X$ is the functional

$$
\begin{aligned}
\mathcal{V}:(-\epsilon, \epsilon) & \longrightarrow \mathbb{R} \\
t & \longmapsto \mathcal{V}(t)=\frac{1}{n+1} \int_{M}\left\langle X_{t}, N_{t}\right\rangle d M_{t},
\end{aligned}
$$

and we say $X$ is volume-preserving if $\mathcal{V}(t)=\mathcal{V}(0)$, for all $t \in(-\epsilon, \epsilon)$.

The next lemma is a well known result and a proof of it can be found in $[6]$.

Lemma 6. Let $x: M^{n} \hookrightarrow \mathbb{R}^{n+1}$ be a closed oriented hypersurface. If $X: M^{n} \times(-\epsilon, \epsilon) \rightarrow \mathbb{R}^{n+1}$ is a variation of $x$, then

$$
\frac{d \mathcal{V}}{d t}=\int_{M} f d M_{t}
$$

In particular, $X$ is volume-preserving if, and only if, $\int_{M} f d M_{t}=0$ for all $t \in(-\epsilon, \epsilon)$.

We can reason as in the proof of Lemma 7 of [10] to get the following:

Lemma 7. Let $x: M^{n} \hookrightarrow \mathbb{R}^{n+1}$ be a closed oriented hypersurface with Gauss map $N$. For any $f \in \mathcal{C}^{\infty}(M)$ satisfying

$$
\int_{M} f d M=0
$$

there exists a volume-preserving variation $X: M^{n} \times(-\epsilon, \epsilon) \rightarrow \mathbb{R}^{n+1}$ of $x$ such that its variational vector field is $f N$. 
For each $k \in\{0, \ldots, n\}$, we define the $(k, F)$-area functional $\mathcal{A}_{k, F}$ : $(-\epsilon, \epsilon) \rightarrow \mathbb{R}$ associated to the variation $X: M^{n} \times(-\epsilon, \epsilon) \rightarrow \mathbb{R}^{n+1}$ by

$$
\mathcal{A}_{k, F}(t)=\int_{M} F\left(N_{t}\right) S_{k} d M_{t} .
$$

Note that for $F \equiv 1$ and $k=0$, it is the classical area functional.

The result below follows from Lemma 3.1 of [14].

Lemma 8. Let $x: M^{n} \hookrightarrow \mathbb{R}^{n+1}$ be a closed oriented hypersurface. If $X: M^{n} \times(-\epsilon, \epsilon) \rightarrow \mathbb{R}^{n+1}$ is a variation of $x$ then

$$
\frac{\partial H_{k+1}^{F}}{\partial t}=\frac{k+1}{b_{k}}\left\{L_{k}(f)+f\left\langle T_{k} \circ d N_{t}, d N_{t}\right\rangle\right\}+\left\langle\left(\frac{\partial X}{\partial t}\right)^{\top}, \nabla H_{k+1}^{F}\right\rangle,
$$

where $f$ is defined in (4.1).

The previous lemma allows us to obtain the first variation of the $(k, F)$-area functional (cf. Theorem 3.3 of $[\mathbf{1 4}])$.

Lemma 9. Let $x: M^{n} \hookrightarrow \mathbb{R}^{n+1}$ be a closed oriented hypersurface. If $X: M^{n} \times(-\epsilon, \epsilon) \rightarrow \mathbb{R}^{n+1}$ is a variation of $x$, then

$$
\mathcal{A}_{k, F}^{\prime}(t)=-b_{k} \int_{M} H_{k+1}^{F} f d M_{t},
$$

where $f$ is defined in (4.1).

Now, motivated by the concept of $(r, s, F)$-linear Weingarten hypersurface, it is natural to consider the variational problem of minimizing the following functional

$$
\begin{aligned}
\mathcal{B}_{r, s, F}:(-\epsilon, \epsilon) & \longrightarrow \mathbb{R} \\
t & \longmapsto \mathcal{B}_{r, s, F}(t)=a_{r} \mathcal{A}_{r, F}(t)+\cdots+a_{s} \mathcal{A}_{s, F}(t)
\end{aligned}
$$

for all variations $X: M^{n} \times(-\epsilon, \epsilon) \rightarrow \mathbb{R}^{n+1}$ of $x: M^{n} \hookrightarrow \mathbb{R}^{n+1}$ which preserve the volume $\mathcal{V}(t)$. The Jacobi functional associated to this variational problem is given by

$$
\begin{aligned}
\mathcal{J}_{r, s, F}:(-\epsilon, \epsilon) & \longrightarrow \mathbb{R} \\
t & \longmapsto \mathcal{J}_{r, s, F}(t)=\mathcal{B}_{r, s, F}(t)+\lambda \mathcal{V}(t),
\end{aligned}
$$

where $\lambda$ is a constant to be determined.

As an immediate consequence of Lemmas 6 and 9 we get

$$
\mathcal{J}_{r, s, F}^{\prime}(t)=\int_{M}\left\{-\sum_{k=r}^{s} a_{k} b_{k} H_{k+1}^{F}+\lambda\right\} f d M_{t} .
$$


In order to make an appropriated choice of $\lambda$, let

$$
\overline{\mathcal{H}}=\frac{1}{\mathcal{A}_{0,1}(0)} \int_{M}\left\{\sum_{k=r}^{s} a_{k} b_{k} H_{k+1}^{F}(0)\right\} d M
$$

be a mean value of the $k$-th anisotropic mean curvatures of $M^{n}, r \leq k \leq s$. We point out to the fact that, in case $\sum_{k=r}^{s} a_{k} b_{k} H_{k+1}^{F}(0)$ is constant, one has

$$
\overline{\mathcal{H}}=\sum_{k=r}^{s} a_{k} b_{k} H_{k+1}^{F}(0)=\sum_{k=r}^{s} a_{k} b_{k} H_{k+1}^{F}
$$

and this notation will be used in what follows without further comments. Hence, choosing $\lambda=\overline{\mathcal{H}}$, we arrive at

$$
\mathcal{J}_{r, s, F}^{\prime}(t)=\int_{M}\left\{-\sum_{k=r}^{s} a_{k} b_{k} H_{k+1}^{F}+\overline{\mathcal{H}}\right\} f d M_{t}
$$

From (4.9) we observe that the critical points of the variational problem described above are exactly the closed $(r, s, F)$-linear Weingarten hypersurfaces. This fact allow us to define a closed $(r, s, F)$-linear Weingarten hypersurface $x: M^{n} \hookrightarrow \mathbb{R}^{n+1}$ being stable when $\mathcal{B}_{r, s, F}^{\prime \prime}(0) \geq 0$, for all volume-preserving variations $X: M^{n} \times(-\epsilon, \epsilon) \rightarrow \mathbb{R}^{n+1}$ of $x$.

Furthermore, we can reason as in [6] to obtain the following stability criterion: a closed $(r, s, F)$-linear Weingarten hypersurface $x: M^{n} \hookrightarrow$ $\mathbb{R}^{n+1}$ is stable if, and only if, $\mathcal{J}_{r, s, F}^{\prime \prime}(0) \geq 0$, for all $f \in \mathcal{C}^{\infty}(M)$ such that $\int_{M} f d M=0$.

The sought formula for the second variation of $\mathcal{J}_{r, s, F}$ is a straightforward consequence of Lemmas 8 and 9 .

Proposition 1. Let $x: M^{n} \hookrightarrow \mathbb{R}^{n+1}$ be closed $(r, s, F)$-linear Weingarten hypersurface. If $X: M^{n} \times(-\epsilon, \epsilon) \rightarrow \mathbb{R}^{n+1}$ is a variation of $x$, then $\mathcal{J}_{r, s, F}^{\prime \prime}(0)$ is given by

$$
\mathcal{J}_{r, s, F}^{\prime \prime}(0)(f)=-\sum_{k=r}^{s}(k+1) a_{k} \int_{M}\left\{L_{k}(f)+\left\langle T_{k} \circ d N, d N\right\rangle f\right\} f d M
$$

for $f \in \mathcal{C}^{\infty}(M)$. 
Proof: From (4.9) we obtain

$$
\begin{aligned}
\mathcal{J}_{r, s, F}^{\prime \prime}(0)= & \left.\frac{\partial}{\partial t}\left(\int_{M}\left\{-\sum_{k=r}^{s} a_{k} b_{k} H_{k+1}^{F}+\overline{\mathcal{H}}\right\} f d M_{t}\right)\right|_{t=0} \\
= & -\int_{M} \sum_{k=r}^{s} a_{k} b_{k}\left(\left.\frac{\partial H_{k+1}^{F}}{\partial t}\right|_{t=0}\right) f d M \\
& +\left.\int_{M}\left(-\sum_{k=r}^{s} a_{k} b_{k} H_{k+1}^{F}+\overline{\mathcal{H}}\right) \frac{\partial}{\partial t}\left(f d M_{t}\right)\right|_{t=0} .
\end{aligned}
$$

Consequently, taking into account (4.4) and (4.8), we get

$$
\begin{aligned}
& \mathcal{J}_{r, s, F}^{\prime \prime}(0)=-\sum_{j=r}^{s}(k+1) a_{k} \int_{M}\left\{L_{k}(f)+\left\langle T_{j} \circ d N, d N\right\rangle f\right\} f d M \\
& -\int_{M}\langle\left(\frac{\partial X}{\partial t}\right)^{\top}, \underbrace{\nabla\left(\sum_{k=r}^{s} a_{k} b_{k} H_{k+1}^{F}\right)}_{0}\rangle f d M \\
& =-\sum_{k=r}^{s}(k+1) a_{k} \int_{M}\left\{L_{k}(f)+\left\langle T_{k} \circ d N, d N\right\rangle f\right\} f d M .
\end{aligned}
$$

To finish the proof, we observe that the above expression depends only on the hypersurface $x: M^{n} \hookrightarrow \mathbb{R}^{n+1}$ and on the function $f \in \mathcal{C}^{\infty}(M)$.

\section{Proof of Theorem 1}

In what follows, we will consider the set

$$
\mathcal{G}=\left\{f \in \mathcal{C}^{\infty}(M): \int_{M} f d M=0\right\} .
$$

If $x(M)$ is (up to translations and homotheties) the Wulff shape of $F$, from the proof of Theorem 1.3 in [14] we have that

$-\int_{M}\left\{L_{k}(f)+\left\langle T_{k} \circ d N, d N\right\rangle f\right\} f d M \geq 0, \quad \forall f \in \mathcal{G} \quad$ and $\quad 0 \leq k \leq n$.

So, from $(4.10), \mathcal{J}_{r, s, F}^{\prime \prime}(f) \geq 0$ for all $f \in \mathcal{G}$. Therefore, $x$ is stable.

Reciprocally, supposing that $x$ is stable, we have $\mathcal{J}_{r, s, F}^{\prime \prime}(f) \geq 0$, for all $f \in \mathcal{G}$. Moreover, from Lemma 3 we can choose

$$
f=\gamma F(N)+\xi\langle x, N\rangle
$$


as the test function, where

$$
\gamma=\frac{\int_{M} F \sum_{k=r}^{s} a_{k} b_{k} H_{k}^{F} d M}{\int_{M} F(N) d M} \quad \text { and } \quad \xi=\sum_{k=r}^{s} a_{k} b_{k} H_{k+1}^{F}=\text { constant. }
$$

We also note that, from (3.3), (3.4), and Proposition 1 we have that

$$
\mathcal{J}_{r, s, F}^{\prime \prime}(0)(f)=-\int_{M} f R_{r, s}[f] d M \geq 0 .
$$

On the other hand, from (3.5) and (3.6) we get

$$
\begin{aligned}
R_{r, s}[f]= & \sum_{k=r}^{s}(k+1) a_{k} I_{k}[f]=\sum_{k=r}^{s}(k+1) a_{k} I_{k}[\gamma F(N)+\xi\langle x, N\rangle] \\
= & \sum_{k=r}^{s}(k+1) a_{k}\left(\gamma I_{k}[F(N)]+\xi I_{k}[\langle x, N\rangle]\right) \\
= & \sum_{k=r}^{s}(k+1) a_{k}\left[\gamma \left(-\left(\begin{array}{c}
n \\
k+1
\end{array}\right)\left\langle\nabla H_{k+1}^{F}, \nabla_{S^{n}} F\right\rangle\right.\right. \\
& \left.+n\left(\begin{array}{c}
n \\
k+1
\end{array}\right) H_{1}^{F} H_{k+1}^{F}-(k+2)\left(\begin{array}{c}
n \\
k+2
\end{array}\right) H_{k+2}^{F}\right) \\
& +\xi\left(\begin{array}{c}
n \\
\left.\left.-\left(\begin{array}{c}
n \\
k+1
\end{array}\right)\left\langle\nabla H_{k+1}^{F}, x^{\top}\right\rangle-(k+1)\left(\begin{array}{c}
n \\
k+1
\end{array}\right) H_{k+1}^{F}\right)\right] .
\end{array}\right.
\end{aligned}
$$

Hence, we get

(5.1) $R_{r, s}[f]=\sum_{k=r}^{s} a_{k} b_{k}\left[\gamma\left(n H_{1}^{F} H_{k+1}^{F}-(n-k-1) H_{k+2}^{F}\right)-\xi(k+1) H_{k+1}^{F}\right]$.

Furthermore, from Lemma 5 we also have that

$$
\begin{aligned}
\mathcal{J}_{r, s, F}^{\prime \prime}(0)(f) & =-\int_{M} f R_{r, s}[f] d M \\
& =-\int_{M}(\gamma F+\xi\langle x, N\rangle) R_{r, s}[f] d M \\
& =-\int_{M}\left(\gamma F R_{r, s}[f]+\xi f R_{r, s}[\langle x, N\rangle]\right) d M
\end{aligned}
$$

But, from (5.1) and Lemma 4 we have $\gamma F R_{r, s}[f]=\sum_{k=r}^{s} a_{k} b_{k} F\left[\gamma^{2}\left(n H_{1}^{F} H_{k+1}^{F}-(n-k-1) H_{k+2}^{F}\right)-\gamma \xi(k+1) H_{k+1}^{F}\right]$. 
Consequently, we get

$$
\begin{aligned}
\gamma F R_{r, s}[f]=\sum_{k=r}^{s} a_{k} b_{k} F[ & \gamma^{2}\left((n-k-1) H_{1}^{F} H_{k+1}^{F}+(k+1) H_{1}^{F} H_{k+1}^{F}\right. \\
& \left.\left.-(n-k-1) H_{k+2}^{F}\right)-\gamma \xi(k+1) H_{k+1}^{F}\right] \\
=\sum_{k=r}^{s} a_{k} b_{k} F\{ & \gamma^{2}(n-k-1)\left[H_{1}^{F} H_{k+1}^{F}-H_{k+2}^{F}\right] \\
& \left.+\gamma^{2}(k+1) H_{1}^{F} H_{k+1}^{F}-\gamma \xi(k+1) H_{k+1}^{F}\right\} .
\end{aligned}
$$

Moreover,

$$
\begin{aligned}
\xi f R_{r, s}[\langle x, N\rangle] & =\xi f \sum_{k=r}^{s}(k+1) a_{k} I_{k}[\langle x, N\rangle] \\
& =\xi f \sum_{k=r}^{s} a_{k} b_{k}\left(-\left\langle\nabla H_{k+1}^{F}, x^{\top}\right\rangle-(k+1) H_{k+1}^{F}\right) \\
& =-\xi f \sum_{k=r}^{s} a_{k} b_{k}(k+1) H_{k+1}^{F} \\
& =-\sum_{k=r}^{s} a_{k} b_{k}(k+1) \xi(\gamma F+\xi\langle x, N\rangle) H_{k+1}^{F} .
\end{aligned}
$$

Therefore, from (5.2), (5.3), (5.4) we have

$$
\begin{aligned}
& \mathcal{J}_{r, s, F}^{\prime \prime}(0)(f)=-\sum_{k=r}^{s} a_{k} b_{k} \int_{M}[ F\left\{\gamma^{2}(n-k-1)\left[H_{1}^{F} H_{k+1}^{F}-H_{k+2}^{F}\right]\right. \\
&\left.+\gamma^{2}(k+1) H_{1}^{F} H_{k+1}^{F}-\gamma \xi(k+1) H_{k+1}^{F}\right\} \\
&\left.-(k+1) \xi[\gamma F+\xi\langle x, N\rangle] H_{k+1}^{F}\right] d M \\
&=-\sum_{k=r}^{s} a_{k} b_{k} \gamma^{2}(n-k-1) \int_{M} F\left(H_{1}^{F} H_{k+1}^{F}-H_{k+2}^{F}\right) d M-\sum_{k=r}^{s} a_{k} b_{k} \int_{M}\left[F\left\{\gamma^{2}(k+1) H_{1}^{F} H_{k+1}^{F}-\gamma \xi(k+1) H_{k+1}^{F}\right\}\right. \\
&\left.-(k+1) \xi[\gamma F+\xi\langle x, N\rangle] H_{k+1}^{F}\right] d M .
\end{aligned}
$$


Thus, from Lemma 3 we get

$$
\begin{aligned}
\mathcal{J}_{r, s}^{\prime \prime}(0)(f)= & -\sum_{k=r}^{s} a_{k} b_{k} \gamma^{2}(n-k-1) \int_{M} F\left(H_{1}^{F} H_{k+1}^{F}-H_{k+2}^{F}\right) d M \\
& -\sum_{k=r}^{s} a_{k} b_{k} \int_{M} F\left\{\gamma^{2}(k+1) H_{1}^{F} H_{k+1}^{F}-\gamma \xi(k+1) H_{k+1}^{F}\right. \\
(5.5) & \left.-(k+1) \xi \gamma H_{k+1}^{F}+(k+1) \xi^{2} H_{k}^{F}\right\} d M \\
= & -\sum_{k=r}^{s} a_{k} b_{k} \gamma^{2}(n-k-1) \int_{M} F\left(H_{1}^{F} H_{k+1}^{F}-H_{k+2}^{F}\right) d M \\
& -\sum_{k=r}^{s} a_{k} b_{k}(k+1) \int_{M} F\left\{H_{1}^{F} H_{k+1}^{F} \gamma^{2}-2 \xi H_{k+1}^{F} \gamma+\xi^{2} H_{k}^{F}\right\} d M .
\end{aligned}
$$

Now, for each point $p \in M^{n}$, we consider the polynomial in $z$ given by

$$
\mathcal{P}_{k, p}(z)=H_{1}^{F} H_{k+1}^{F} z^{2}-2 \xi H_{k+1}^{F} z+\xi^{2} H_{k}^{F} .
$$

In this case, we have that the discriminant of $\mathcal{P}_{k, p}$ is

$$
\Delta=4 \xi^{2}\left[\left(H_{k+1}^{F}\right)^{2}-H_{1}^{F} H_{k+1}^{F} H_{k}^{F}\right]=4 \xi^{2}\left(H_{k+1}^{F}\right)^{2}\left(1-\frac{H_{1}^{F} H_{k}^{F}}{H_{k+1}^{F}}\right) \leq 0,
$$

where the last inequality in the expression above is given by Lemma 1 . Thus, we have that $\mathcal{P}_{k, p} \geq 0, \forall p \in M^{n}$, and $\forall k \in\{r, \ldots, s\}$. In particular,

$$
H_{1}^{F} H_{k+1}^{F} \gamma^{2}-2 \xi H_{k+1}^{F} \gamma+\xi^{2} H_{k}^{F}=\mathcal{P}_{k, p}(\gamma) \geq 0,
$$

$\forall p \in M^{n}$ and $\forall k \in\{r, \ldots, s\}$. So, from (5.5), (5.6), and Lemma 1, we have

$$
\begin{aligned}
\mathcal{J}_{r, s}^{\prime \prime}(0)(f)= & -\sum_{k=r}^{s} a_{k} b_{k} \gamma^{2}(n-k-1) \int_{M} F\left(H_{1}^{F} H_{k+1}^{F}-H_{k+2}^{F}\right) d M \\
& -\sum_{k=r}^{s} a_{k} b_{k}(k+1) \int_{M} F \mathcal{P}_{k, p}(\gamma) d M \leq 0 .
\end{aligned}
$$

From (5.7), since $x$ is stable and taking into account that each therm of $\mathcal{J}_{r, s}^{\prime \prime}(0)(f)$ is nonpositive, we easily see that they are, in fact, identically zero. Consequently, using once more (5.7), we get that $H_{1}^{F} H_{k+1}^{F}-$ $H_{k+2}^{F}=0$ on $M^{n}$, for all $r \leq k \leq s$. So, from item (ii) of Lemma 1 , we have that the anisotropic principal curvatures are equal. Therefore, Lemma 2 assures us that, up to translations and homotheties, $x(M)$ is the Wulff shape of $F$, finishing the proof of Theorem 1. 
Remark 2. Concerning our constraint on the sign of $H_{s+1}^{F}$ in Theorem 1 , we observe that it is crucial to the success of our technique, since the Gardin type inequalities of Lemma 1 constitutes our algebraic tool to detect the anisotropic umbilicity of the closed $(r, s, F)$-linear Weingarten hypersurface. We also note that this phenomenon already occurs in the context of the works of Alencar-do Carmo-Rosenberg [1] and BarbosaColares $[6]$.

\section{Acknowledgements}

The second author is partially supported by CNPq, Brazil, grant 303977/2015-9. The third author is partially supported by CNPq, Brazil, grant 308757/2015-7. The authors would like to thank the referee for her/his valuable suggestions and useful comments which improved the paper.

\section{References}

[1] H. Alencar, M. do Carmo, and H. Rosenberg, On the first eigenvalue of the linearized operator of the $r$ th mean curvature of a hypersurface, Ann. Global Anal. Geom. 11(4) (1993), 387-395. DOI: $10.1007 / \mathrm{BF} 00773553$.

[2] C. P. Aquino, H. F. de lima, and M. A. L. Velásquez, A new characterization of complete linear Weingarten hypersurfaces in real space forms, Pacific J. Math. 261(1) (2013), 33-43. DOI: 10.2140/pjm. 2013.261.33.

[3] C. P. Aquino, H. F. de Lima, and M. A. L. Velásquez, Generalized maximum principles and the characterization of linear Weingarten hypersurfaces in space forms, Michigan Math. J. 63(1) (2014), 27-40. DOI : $10.1307 / \mathrm{mmj} / 1395234357$.

[4] J. L. Barbosa and M. Do Carmo, Stability of hypersurfaces with constant mean curvature, Math. Z. 185(3) (1984), 339-353. DOI: $10.1007 / \mathrm{BF} 01215045$.

[5] J. L. Barbosa, M. do Carmo, and J. Eschenburg, Stability of hypersurfaces of constant mean curvature in Riemannian manifolds, Math. Z. 197(1) (1988), 123-138. DOI: 10.1007/BF01161634.

[6] J. L. M. Barbosa And A. G. Colares, Stability of hypersurfaces with constant $r$-mean curvature, Ann. Global Anal. Geom. 15(3) (1997), 277-297. DOI: 10.1023/A:1006514303828.

[7] A. Barros, J. Silva, and P. Sousa, Rotational linear Weingarten surfaces into the Euclidean sphere, Israel J. Math. 192(2) (2012), 819-830. DOI: $10.1007 / \mathrm{s} 11856-012-0053-9$.

[8] H. Chen And X. Wang, Stability and eigenvalue estimates of linear Weingarten hypersurfaces in a sphere, J. Math. Anal. Appl. 397(2) (2013), 658-670. DOI: 10.1016/j.jmaa.2012.08.003. 
[9] U. Clarenz, The Wulff shape minimizes an anisotropic Willmore functional, Interfaces Free Bound 6(3) (2004), 351-359. DOI: 10.4171/IFB/104.

[10] A. G. Colares and J. F. DA Silva, Stable hypersurfaces as minima of the integral of an anisotropic mean curvature preserving a linear combination of area and volume, Math. Z. 275(1-2) (2013), 595-623. DOI : 10.1007/s00209-013-1149-6.

[11] J. F. DA Silva, H. F. De Lima, and M. A. L. Velásquez, The stability of hypersurfaces revisited, Monatsh. Math. 179(2) (2016), 293-303. DOI : 10.1007/s00605-015-0776-x.

[12] G. H. Hardy, J. E. Littlewood, And G. Pólya, "Inequalities", Reprint of the 1952 edition, Cambridge Mathematical Library, Cambridge University Press, Cambridge, 1988.

[13] Y. HE AND H. LI, A new variational characterization of the Wulff shape, Differential Geom. Appl. 26(4) (2008), 377-390. DOI: 10.1016/j.difgeo.2007.11.030.

[14] Y. HE AND H. LI, Stability of hypersurfaces with constant $(r+$ 1)-th anisotropic mean curvature, Illinois J. Math. 52(4) (2008), 1301-1314.

[15] Y. J. HE AND H. Z. LI, Integral formula of Minkowski type and new characterization of the Wulff shape, Acta Math. Sin. (Engl. Ser.) 24(4) (2008), 697-704. DOI: 10.1007/s10114-007-7116-60.

[16] M. Koiso And B. Palmer, Geometry and stability of surfaces with constant anisotropic mean curvature, Indiana Univ. Math. J. 54(6) (2005), 1817-1852. DOI: 10.1512/iumj.2005.54.2613.

[17] M. KoIso AND B. PALmer, Stability of anisotropic capillary surfaces between two parallel planes, Calc. Var. Partial Differential Equations 25(3) (2006), 275-298. DOI: 10.1007/s00526-005-0336-7.

[18] M. Koiso And B. PAlmer, Anisotropic capillary surfaces with wetting energy, Calc. Var. Partial Differential Equations 29(3) (2007), 295-345. DOI : 10.1007/s00526-006-0066-5.

[19] M. Koiso And B. Palmer, Anisotropic umbilic points and Hopf's theorem for surfaces with constant anisotropic mean curvature, Indiana Univ. Math. J. 59(1) (2010), 79-90. DOI: 10.1512/iumj . 2010. 59.4164.

[20] H. Li, Y. J. SuH, AND G. WEI, Linear Weingarten hypersurfaces in a unit sphere, Bull. Korean Math. Soc. 46(2) (2009), 321-329. DOI : $10.4134 /$ BKMS .2009.46.2.321.

[21] M. M.-C. LI, On complete stable minimal surfaces in 4-manifolds with positive isotropic curvature, Proc. Amer. Math. Soc. 140(8) (2012), 2843-2854. DOI : 10.1090/S0002-9939-2011-11113-4. 
[22] M. J. Micallef And J. D. Moore, Minimal two-spheres and the topology of manifolds with positive curvature on totally isotropic two-planes, Ann. of Math. (2) 127(1) (1988), 199-227. DOI: 10.2307/1971420.

[23] B. Palmer, Stability of the Wulff shape, Proc. Amer. Math. Soc. 126(12) (1998), 3661-3667. DOI: 10.1090/S0002-9939-98-04641-3.

[24] R. C. REILly, Variational properties of functions of the mean curvatures for hypersurfaces in space forms, J. Differential Geometry 8 (1973), 465-477.

[25] R. C. REILly, The relative differential geometry of nonparametric hypersurfaces, Duke Math. J. 43(4) (1976), 705-721.

[26] S. SHu, Linear Weingarten hypersurfaces in a real space form, Glasg. Math. J. 52(3) (2010), 635-648. DOI: 10.1017/S001708951 0000480.

[27] J. E. TAylor, Crystalline variational problems, Bull. Amer. Math. Soc. 84(4) (1978), 568-588. DOI : 10.1090/S0002-9904-1978-14499-1.

[28] J. E. Taylor, J. W. Cahn, and C. A. Handwerker, Geometric models of cristal growth, Acta Metall. Mater. 40(7) (1992), 1443-1474. DOI: 10.1016/0956-7151 (92)90090-2.

[29] M. A. Velásquez, A. F. De Sousa, and H. F. de Lima, On the stability of hypersurfaces in space forms, J. Math. Anal. Appl. 406(1) (2013), 134-146. DOI: 10.1016/j.jmaa.2013.04.045.

[30] S. Winklmann, A note on the stability of the Wulff shape, Arch. Math. (Basel) 87(3) (2006), 272-279. DOI: 10.1007/s00013-0061685-y.

Jonatan F. da Silva:

Departamento de Matemática

Universidade Federal do Ceará

60455760 Fortaleza, Ceará

Brazil

E-mail address: jonatanfloriano@mat.ufc.br

Henrique F. de Lima and Marco Antonio L. Velásquez:

Departamento de Matemática

Universidade Federal de Campina Grande

58429-970 Campina Grande, Paraíba

Brazil

E-mail address: henrique@dme.ufcg.edu.br

E-mail address: marco.velasquez@pq.cnpq.br

Primera versió rebuda el 5 d'abril de 2016,

darrera versió rebuda el 6 d'octubre de 2016. 